\title{
Development of Model for Estimation of Age Group of Drivers by using Soft Computing Tools \\ ${ }^{1}$ Sanjay $S$ Wankhede, ${ }^{2}$ Preeti Bajaj \\ ${ }^{1}$ Research Scholar, Department of Electronics Engineering, G.H. Raisoni College of Engineering, Nagpur Email: ${ }^{1}$ sanjay.wankhede@ raisoni.net ${ }^{2}$ preeti.bajaj@raisoni.net
}

\section{Received: 09 ${ }^{\text {th }}$ July 2018, Accepted: $14^{\text {th }}$ August 2018, Published: $31^{\text {st }}$ August 2018}

\begin{abstract}
Road accidents are rapidly increased day to day, that affects the various humans' life. Driver is the main parameter of the vehicle that sources the accidents. Many researchers interested to gather the data of the people who are accountable for the accidents. These papers mainly focus on the age group of the driver because age group is the most important factor for the driver's behaviour. The age group estimation of the driver is based on his or her facial extraction mainly on the basis of multidimensional facial images. In this paper, we developed neural network model to analyze the age group of the driver using facial features extraction.
\end{abstract}

Keywords: Video, Frame, Face Detection, Neural Network, Age Estimation

\section{Introduction}

The road accidents occurs because driver behaviour, vehicle failure, roadway design and poor roadway maintenance. Over $90 \%$ of road traffic accidents involve human factor, there were major effects of age, experience and education on violation behaviour of traffic rules [1]. Those people who are really interested to collect the data for the people who are responsible for the accident or who are suffer from the accidents, this paper will really help them. Age group is also key parameter for the driver's data collection. In this paper Age group of drivers is estimated. Automatic age estimation has rarely been explored. In a lot of behaviour of a human being the age development affects organization, size and look. Some characteristics of craniofacial morphology become visible only in human being of certain age range and transform during the aging. From birth to later life Changes in skin texture generally take place. According to Geng et al [2] Most of the change are cause by change in facial structures development like Cheeks are spread over a larger area, Chin becomes more prominent, reduces the free space on the surface of the skull. In addition to changes caused by facial structures development, some small changes in the skin take place, Facial hair turn out to be denser and change colour, Skin colour changes, Skin become darker, thinner, fewer flexible and more tough. Moreover, wrinkles, hang down cheeks and lowered bags under the eyes become visible, but most of the facial structures development occurs at an early age of the individual. [3]. And if we consider all face then all have dissimilar features for example some male have beard and mustache but some male didn't have. Some male haveshort hair but some female also have short hair. There are many lacunas so that's why we can't justify the accurate age of the person from their face. For that many researcher are distribute their ages in some group such as child, young, youth and old. Or they do this work in the groups. In this paper 10 group is distributed as like 1-10, 11-15, 16-22, 23-27, 28-35, 3642 , 43-50, 51-58, 59-65, and 67-80. No one can give the precise accuracy hence our aim is to minimize the age group, for that larger data base is required for training the neural network. There are many ways to estimate the age group of the drivers. Some researcher uses the landmark method. In which they use the landmark points on the face. In that some uses the 68 point or some use the 57 points on the face, is all up to the researchers. And in FGNET database they have some extracted feature. Some researcher manipulate the ratio method in that they utilize the ratio between the distance of the eyes and nose, and eye and mouth, or the ratio of the distance between mouth and nose and distance between the nose and eye and many more[4]. And some researcher calculates the distance between the eye and distance between the eye and nose. In that they have their individual reference parameters. And by using those parameters they calculate the age of person. But all they are facing with the same problem because of the insufficient data base, accurate age is not estimated

\footnotetext{
Related Work

Many researchers have already done the Collection of traffic videos and faces detection work. Guillaume Dave et al [5] interrogate various designs for face detection system on mobile phones. In this paper, Colour partitioning and pattern matching for face detection technique is used. Lots of limitation in the algorithm is still there to detect the face. If skin colour is same as that of the background colour of an image, algorithm can't analyze. In the pattern matching algorithms obtained results are reliant on the standard pattern chosen, brightness condition etc. Pattern matching algorithms not perform satisfactory while detecting faces of indigenous origins. To achieve better results, eigen face and fisher face algorithms
} 
are developed for face recognition. After implementation of the face recognition algorithm on DROID phone, integrate it with standard face detection application for testing the whole face recognition system. This methodology likes to analyze better results for face detection and recognition.

Devendra singh and Dhreej[6] used RGB, YCbCr, CEILAB $\left(\mathrm{L}^{*} \mathrm{a} * \mathrm{~b}\right)$ and HSV colour models for skin colour segmentation, colour model is a system for creating full range of colour from small set of primary colour. For skin colour pixels firstly they convert the colour image into gray scale image for that they use order such as Red>95.Green>40and Blue $>20$, by applying such condition they find the skin colour and from that they detect face. They firstly do Skin colour segmentation, then conversion into Binary image then Rejection of non-face regions. For that they utilize the bounding box property and the hole number here we know that every face doesn't contain the skin colour, two eye, and lips having the different colour hence they look like black colour in binary image hence that called hole number, And by using those properties finally they detect the face from the given image. Those two methods are use for the face detection. The proposed Algorithm for this detects only human faces in an image.

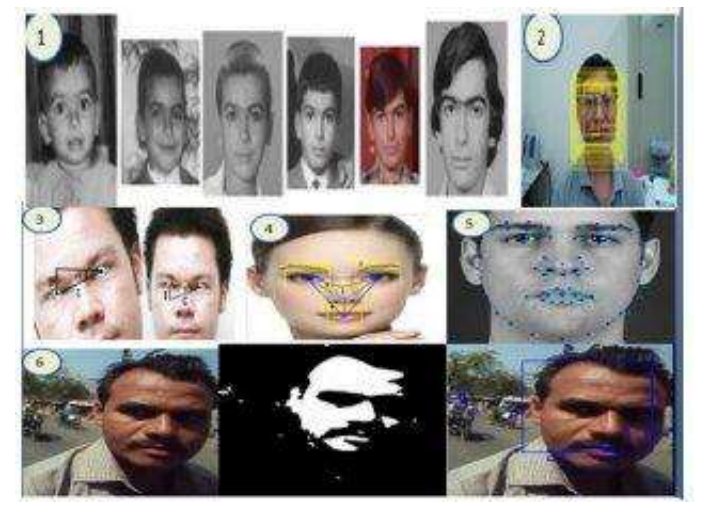

Figure 1.Age Estimation Study on Different Images

In figure 1,1 . Shows the sample image of same person in different age [7], 2. Shows the bounding box technique for detecting the face [7], 3. Shows the technique from which Age is calculated here distance between two eyes is considered, 4 shows the ratio method for estimating the age,5. Shows the landmark points method for estimate age [8], and 6. Show how frame is converted into binary image and face is detected from frame.

For the age estimation, Nabil Hewahi et al [8] use the neural network and they manipulate database from the MORPH and FGNET but because of insufficient data base they didn't give the correct accuracy hence they distributed the age in some groups such 1-12, 13$25,2645,46-63$, for that they use the landmark technique, They use the 68 landmarks point on the skin and by using those landmarks find the age group, Petra GRD [3] use the ratio method for estimation the age group, they also use the data base from the MORPH, and FGNET .In this paper ,author uses the distance ratio between the eyes-nose and eye-lips and so on. By using such methods they estimate the age group of the persons.

Kwon and Lobo [9] proposed the analytics for age analysis from facial images. Analysis is based on facial structure and wrinkle. One of the researcher Patterson et al[10] propose Active appearance model (AAM) based on the principal component analysis method.

\section{Methodology}

Firstly video of the traffic is collected then by framing it face is detected from frame and then from face of the driver age group is estimated. For that video is collected from the signal areas because from the signal there are many drivers drive their vehicle in slow speed so that's why image of the drivers is capture perfectly. For finding the face, Skin Colour method is use in this paper. For that some condition apply like firstly colour image transformed into RGB image then applying some colour order image is converted into binary image and then face is detected. And from Face, Age group of drivers is estimated; Age group is a number of people classed together as being of similar age. Here for estimating age group of person artificial neural network is use. and neural network also used in character recognition, future state prediction like many problems, For accumulating the image of faces FGNET and MORPH database is followed, database is a structure set of data held in computer, especially one system that is accessible in various way, FGNET and MORPH consist of more than 1000 facial images in that FGNET contain already extracted feature. So there is insufficient images collection, hence some image is taken from the camera and net, in these paper 200 images is taken to train the neural network. All images carry the face of person having the different Age group. Age estimation is a challenging issue emerged in past few years on the basis of facial exaction. Many of the researcher are already examine this work, they also collect the database and estimate the age group of person from their face. But they didn't give the precise age. To estimate the age is very hard task. So till now, no one can find the accurate age from the face. In this paper some pixel parameter is measured. Here 256 pixel points is selected and from 256 pixel values many parameters such as Kurtosis, Mean, Variance, Skewnes is calculated. And for the 
datasheet with those 260 value F1, F2 and F3 is also count to the input, Here F1, F2 and F3 are,

$\mathrm{F} 1=$ distance between the left eye to right eye/ distance between eye to nose.

$\mathrm{F} 2=$ distance between the left eye to right eye/distance between eye to lips.

F3= distance between eye to nose/distance between eye to lips.

These 263 values operate to train the neural network. And from this values age of driver is estimated. This will help to control over the traffic violence and apart from traffic violence there are many uses of this technique such as for finding the age group of Criminals and in medical purpose also for finding the age group of unknown dead body.

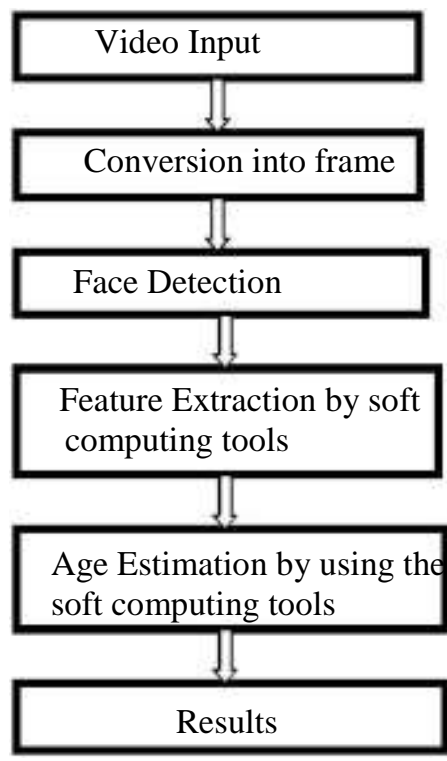

Figure 2. Flow Chart of Work

\section{a. Video Input}

Here first is video input which is taken from the traffic mainly the video is taken from the signal areas because their vehicles are slow in speed, and that will help to collect the proper video in which face of the driver is noticed clearly.

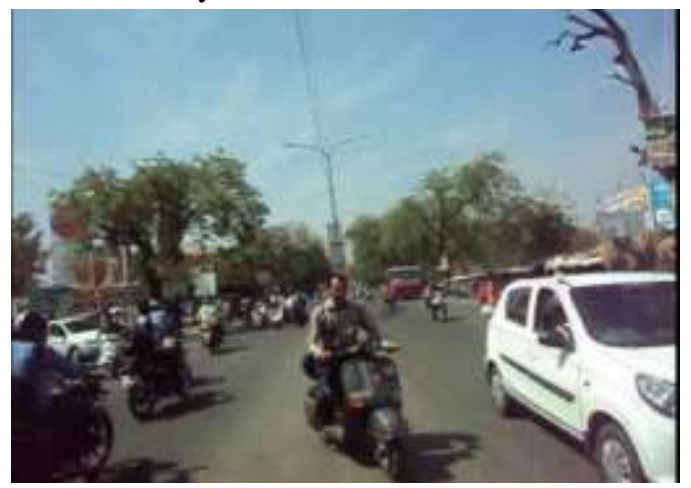

Figure 3. Video Input

\section{b. Frame Conversation}

Transform that image into frame. Frame should select such a way, from where face of the driver will notice clearly.

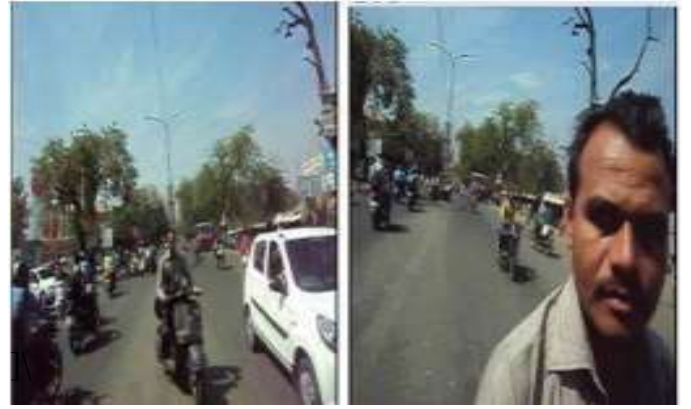

Figure 4. Frame Conversion from Video

\section{c. Face Detection}

For face detection, skin colour is taken to be appreciable, here some order is apply for skin colour

1. Red $>92$.

2. Blue $>20$.

3. Green $>40$.

4. $(\mathrm{R}-\mathrm{G})>15$.

If all the above condition is satisfy then there is 1 and if not then 0 here 1 represent the white colour and the 0 represent the black colour.
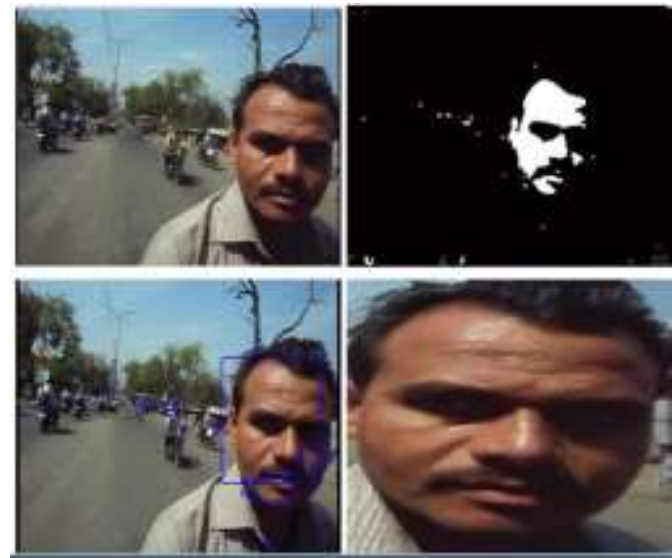

Figure 5. Face Detected From Frame

In that background is of Black color and the face is White color. Now by using the Holes Number. Here for the hole, firstly use bounding box property. Here the rectangular box which is of certain range having the Skin color that is the face. Now we all know every face does not contain the skin color. Our two Eye and Lips are having the different color. So that they represent by the black color we will call them the Holes. Now the rectangular box having the skin color with minimum two holes that box called the face. Fig 5 shows the face, 
by using such a condition face of driver is detected in the MATLAB. And further Age group is estimated.

\section{d. Age Estimation}

Estimating means calculating or predicting something from the artificial computers that called the estimation. Conversion into frame For calculating the age from the face, the facial database is required for that the facial image is choose from the MORPH, FGNET and because of insufficient data collect some image from the net. For training neural network here 263 parameter is given as an input to the neural network Pixel.

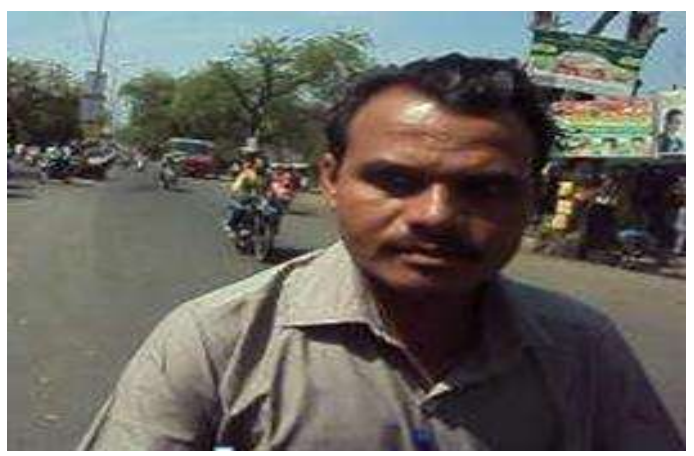

Figure 6. Input Image of Driver

\section{Experimental Result}

\section{Pixel Value}

Every image contain some pixel values from there histogram. Here 256 pixel values is taken as an input to the neural network.

Table 1. Data sheet of pixel value of input image of drivers

\section{Image Parameters}

Here parameters are taken from that pixel values that are mean variance kurtosis and Skewnes, Skewnes is

\begin{tabular}{|c|c|c|c|c|c|c|c|c|c|c|c|c|c|c|c|c|}
\hline & 1 & & $\zeta$ & 0 & E & I & $\varepsilon$ & 1 & & 1 & I & 1 & II & I & $\theta$ & 1 \\
\hline 1 & 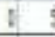 & 3 & 195 & 4]7 & 355 & 157 & 83 & $\pi$ & 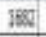 & 125 & 44! & 196 & 1232 & 135) & 797 & \\
\hline I & 8 & & 4II & 379 & 300 & 306 & 804 & 78 & 1361 & 1227 & B5 & 198 & 1538 & 1308 & 711 & \\
\hline i & $E$ & 5) & ES: & 312 & 部 & it & 766 & 도 & IEN & 1146 & B8: & 198 & 138 & 1231 : & 760 & \\
\hline 4 & E. & 4 & $72 x$ & 36 & 3 语 & 20 & 864 & $\mathrm{n}$ & 180 & 태 & $980^{\circ}$ & 150 & $4 \pi$ & 1111 & 7था & \\
\hline 1 & 8 & & $\theta$ & 84 & (⿻) & 301 & $\pi$ & $\pi$ & 154 & 100 & 580 & INE & 1658 & 5.2. & $x$ & \\
\hline t & 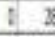 & & 72 & 87 & 327 & 28 & t5 & 44 & 130 & 50 & 950 & 1201 & 1635 & 57: & 712 & \\
\hline 7 & \& 12 & & 12 & 26 & 45 & 158 & 72. & $\pi$ & 115: & 97 & 92 & 128 & 14: & $B E$ & Eस] & \\
\hline 1 & 1 & & 75: & 24 & 34 & 12 & 42 & (2) & 11 혀 & $4:$ & 20. & 1318 & 1835 & 82 & 60 & \\
\hline 1 & t. & & 68 & 273 & 촐ㄹ & 48 & $(60)$ & 65 & 1225 & प्र: & $92 \pi$ & 1255 & $15 \pm \sqrt{1}$ & 结 & 424 & \\
\hline 2 & 2 & & तit: & $2 \pi$ & 354 & 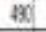 & 65 & 68 & $15 \mathrm{H}$ & B1. & 580 & 1275 & 1502 & ㅂ! & 56. & \\
\hline 11 & I II & & 114 & $\mathrm{XS}$ & 32 & ne & 206 & 72. & 120 & 500 & 58) & 1200 & Wys & $w$ & 54 & \\
\hline a & 1. 18 & & 574 & (2) & 急 & 45: & 74] & 80 & $12 \pi$ & 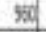 & 920 & (25) & 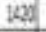 & 635. & 221 & \\
\hline 3. & 158 & & $x a$ & 311. & 30 & $5 \times$ & 143 & 96 & 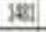 & BD. & $1 \times 3$ & 1123 & 158 & EF & 4 & \\
\hline y & 8121 & & 1560 & $x 5$ & 304 & $5 \pi$ & 34 & I81] & 13 & $t 5$ & $52 \mathrm{~F}$ & 126 & ta: & (6): & E: & \\
\hline 5 & ? 151 & & 139 & 32 & 2⿺辶⿻二丨 & $6 ! 6$ & 34 & 155 & 1115 & 5 5: & $10 \%$ & 124. & $4 \%$ & 72 & 15 & \\
\hline a & 5. 140 & & b8) & 316 & $x$ & 64 & 94 & 164 & 18 & 451 & 1093 & 122 & $1645^{\circ}$ & $B x$ & & \\
\hline
\end{tabular}

Table 1 Show the 256 Pixel Value of Input Image of Drivers Face Which will give the Input to NN. a measure of asymmetry of data, a rounded a sample mean, it's a normal distribution, and Kurtosis is a measure of whether the data are peaked or flat, and it maser the sharpness of curve, Here for Fig. 6. Mean is 254.9531, Variance is 29.7573, Kurtosis is 1.4858 , and Skewnes is -2.16 .

\section{Ratio Parameters}

Ratio parameters taken from the facial image that are F1, F2 and F3. From Fig. 7.

$\mathrm{F} 1=$ distance between the left eye to right eye/ distance between eye to nose. That is 1.1961 ,

$\mathrm{F} 2=$ distance between the left eye to right eye/distance between eye to lips. that is $0.6634, \mathrm{~F} 3=$ distance between eye to nose/distance between eye to lips. That is 0.79698 ,

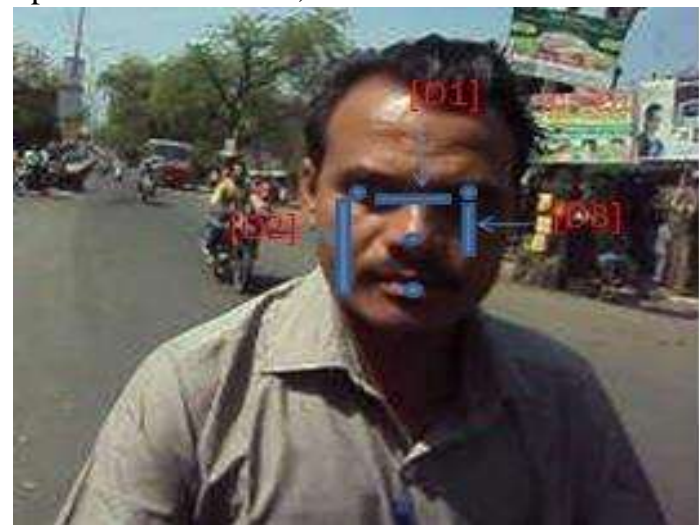

Figure 7 [D1] is Distance between Two Eyes, [D2] is Distance From Eye to Lips and [D3] is Distance from Eye to Nose

Total 263 inputs given to the neural network and age of person are estimated.

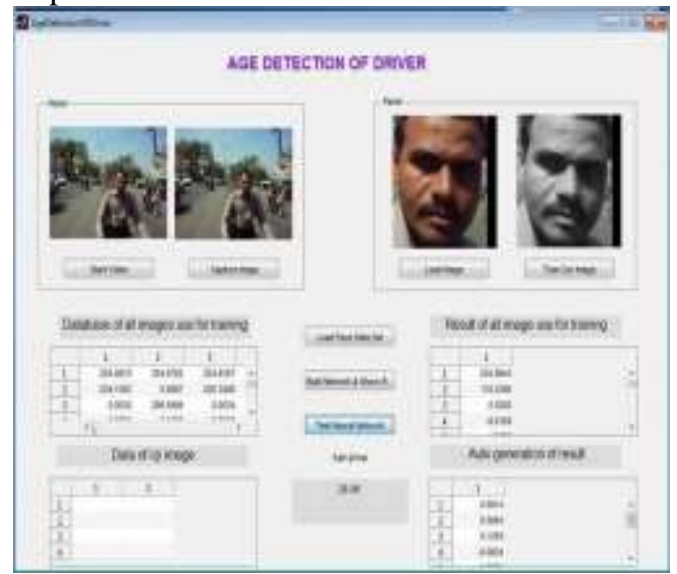

Figure 8. Age Group of Driver is Estimated 
Figure 8 shows the manipulation of age group of driver. In the first block the real time video is considered and it is converted into frames for detecting the human face from captured frame. In the next two blocks all the 263 parameters are trained to calculate the age of human which is shown in the last block.

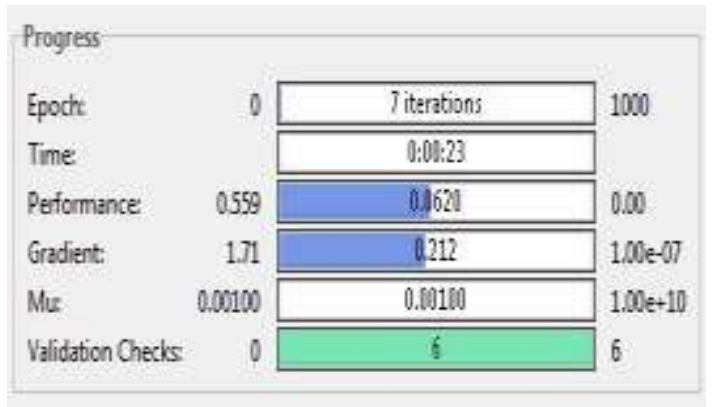

Figure 9. Learning progress of Neural Network.

Experiments are carried out on the database to find the accuracy and efficiency of the proposed algorithm. The database contains 200 images of different age group which are further used to train the neural network for estimating the age as an output.

\section{Conclusion}

Because of road accident increase day to day, which affects the human life, so this paper will help to reduce the road accident. Age group is important characteristic of the driver's behavior, in this paper video of traffic collect two dimensional facial image. For estimation of age group of driver 263 parameters are calculated, that will help to control over the traffic violence.

\section{References:}

1. A study of the psychological factors that influence the rule violation behaviour of drivers (Mohammed Najeeb. P. M Motor Vehicle Department, Ministry of Transport, Kerala, India)

2. Geng Xin, Fu Yun, Smith-Miles Kate. 2010. Automatic Facial Age Estimation, Tutorial at PRICAI 3. Petra GRD "Introduction to human age estimation using face images" research papers faculty of materials science and technology in trnava slovak university of technology in Bratislava,2013

4. Ranjan Jana, Debaleena Datta, and Rituparna Saha "Age Group Estimation using Face Features" International Journal of Engineering and Innovative Technology (IJEIT) Volume 3, Issue 2, August 2013 5. Guillaume Dave, Xing Chao, Kishore Sriadibhatla Department of Electrical Engineering Stanford University Stanford, USA "Face Recognition in Mobile Phones"
5. Devendra Singh Raghuvanshi and Dheeraj Agrawal "Human face detection by using skin color segmentation, face features and regions properties" international journal of computer applications (0975- 8887) volume 38- no.9, january 2012

6. Chin-Teng Lin, Dong-Lin Li,Jian-Hao Lai, Ming-Feng Han and Jyh-Yeong Chang "Automatic age estimation system for face images" International Journal of Advanced Robotic Systems,2012.

7. Nabil Hewahi, Aya Olwan, Nebal Tubeel, Salha EL-Asar, Zeinab Abu-SultanFaculty of Information Technology Islamic University of Gaza - Palestine "Age estimation based on neural networks using face features" journal of emerging trends in computing and information sciences, 2010

8. Young H. Kwon, Niels da Vitoria Lobo. 1999. Age Classification from Facial Images, Computer Vision and Image Understanding, 74(1), pp. 1-21.

9. E. Patterson, K. Ricanek, M. Albert, E. Boone. 2006. Automatic representation of adult aging in facial images. Proceedings of the 6th IASTED International Conference on Visualization, Imaging, and Image Processing, pp.171-176 